\title{
A Review of Bipolar Disorder
}

\author{
Yutong $\mathrm{Wu}^{1, \mathrm{a},{ }^{*}, \dagger}$, Shuo Yin ${ }^{2, \mathrm{~b}, *}, \dagger$ \\ ${ }^{1}$ Saint Paul American School Afflicated to Nacel Internationa School System, Beijing, China \\ ${ }^{2}$ Yaohua High School, Tianjin, China

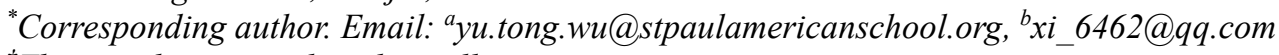 \\ ${ }^{\dagger}$ These authors contributed equally.
}

\begin{abstract}
Bipolar disorder is a mood disorder, and the symptoms of depressive episodes in bipolar disorder are often similar to those of monophasic depression and often clinically indistinguishable. The most characteristic feature of patients with bipolar disorder is the alteration of effect to excessive affective highs or excessive lows. The disease is manic when it manifests high emotion during its onset and depressed when it manifests low emotion. These patients can have multiple episodes repeatedly during their lifetime, and the interval between episodes is intermittent. At this time, the patient's mental state completely returns to normal. After the disease, their mental state rarely becomes declining. This article discussed several elements related to bipolar disorder: influencing factors, including gender, age and family history; and also treatment methods including interpersonal and social rhythm therapy, cognitive-behavioral therapy and psychoeducational interventions. Bipolar disorder is a psychiatric disorder that is difficult to heal fully, and patients who recover with medication also have a high relapse rate within one year after stopping the medication. However, the family and social environment in which one lives is tolerant and is an essential factor in alleviating the condition. Social support from loved ones and inclusion and companionship will provide a favorable healing environment and is a valid predictor of emotional stability afterward.
\end{abstract}

Keywords: bipolar disorder, treatment, predictor, depression.

\section{INTRODUCTION}

There are two-faced people in daily life, but not spies. They are on a roller coaster of emotions: sometimes driving into the lows, they feel sad and desperate; sometimes stepping into the peaks, they feel high and out of control. It is because they are sick, and the name of the disease is bipolar disorder. Bipolar disorder is a genetic disorder in which the patient's mood shifts between extreme excitement or mania and severe depression. At the same time, the patient may have abnormalities in thinking and behavior. In all modern classifications, such as ICD10 or DSM-5, the most important characteristics of bipolar disorder is that it requires the patient to have both a history of depressive episodes and a history of manic episodes $[1,2]$.

According to the data provided by the National Institute of Mental Health (NIMH), annually bipolar disorder has affected around 2.8 percent of the U.S. population [3]. Around 2.4 percent of people worldwide have been diagnosed with bipolar disorder [4]. The prevalence of bipolar disorder have different condition in different area. India have the lowest death rate, which is
0.1 percent. The highest death rate round the world is in U.S. which was once hit 4.4 percent $[3,5]$. According to the data, that bipolar disorder is relatively prevalent. Not only it has a high percentage of morbidity, but also it has a high suicide rate, 80 percent of the people who have bipolar disorder have attempted suicide in their life [6]. Bipolar disorder is a relatively complex mental disorder. It has three main types of bipolar, the level of depression and mania episode are greatly different, including other types of different.

There are a variety of types of disability associated with bipolar disorder: increased suicidal behavior, increased health care use and costs, higher unemployment, higher dependence on public assistance, lower annual income, increased work absenteeism owing to illness decreased work productivity, poorer overall functioning, lower quality of life, and reduced life span [7-9]. In this sense, bipolar disorder causes individual misery and inflicts the whole society with the losing of proper-functioning labor force and extra costs for health care. 


\section{PREDICTORS OF OUTCOME}

\subsection{Gender}

Gender does affect the performance of symptoms for bipolar disorder. For bipolar disorder, that there are two episodes, mania episode, and depression episode. The distribution of the two episodes could be different based on the patent's gender. According to a study that has been done on 111 patients, that female patients show fewer mania episodes, but more depression episodes, compared to male patients. For the transitions of bipolar disorder, based on family disease, a woman and man have a similar chance of being transmitted. The researchers thus concluded that the transition of bipolar disorder is not limited to the $\mathrm{x}$ chromosome [10].

During the treatment of the bipolar disorder, there is a high chance for the patients to gain more weights, mostly due to the drug. According to the instructions from anti-depression drugs, such as Zoloft. Gaining weight is classified as a high chance side effect. In modern society, people care a lot about their body shape, gaining weight is a problem for most people. Gaining weight will decrease a person's self-esteem. They will think that they are no longer charming as before, and that will make the patient anxious. This weight gaining problem is especially severe among women, since most women want to get rid of the fats, and women have a relatively high desire to peruse for beauty. The woman cares more about their body shape than that of man, gaining weight will bring them more stress and feeling of anxious than that of man. Comparing the subjective experience of the prognosis, the woman could suffer more from the side effect of gaining weight, and thus have a higher chance of giving up treatment, or aggregation of the mental disorder. According to a research based on the subjective experience difference on man and woman, the researchers have discovered that due to the negative subjective experience of the bipolar disorder, and during the treatment of bipolar disorder, the patient's cooperation on the treatment might decrease. For female patients, the negative subjective experience was especially on the worries of gaining weight, worries of other side effects of the medicine, and a nigher desire for mental support that comes from the society, compared to male patients, the female patients have been more severely affected by those negative subjective experiences [11].

\subsection{Age}

Generally, the symptoms of bipolar disorder alternate between a depressive state and a manic state. During a depressive episode, the patient's mood is the low brain and action response becomes slower. They become lazy in their daily life and are reluctant to do things or interact with people $[12,16]$. They will go out less often, sit alone, or lie in bed. However, when a manic episode occurs, they will become extraordinarily excited, and their own abilities and hobbies will become stronger [12]. Patients will think of themselves as brainy and responsive. Symptoms also vary by age group. Some studies have shown that older patients with bipolar disorder have a longer latency period for the manic phase compared to younger patients, and far longer than younger patients [12]. In addition, the frequency of mixed episodes is much higher in younger patients than in older patients. The asymptomatic cycle is longer in older patients [13]. At the same time, studies have shown that the likelihood of recurrence of depressive symptoms in the early stages of recovery from bipolar disorder is proportional to the length of the patient's depression or anxiety in the previous year, but not in mania $[14,15]$.

The dangers of bipolar disorder can be described in terms of the patient's life. In terms of work, if an adult has bipolar disorder, some people may feel increased productivity when they are mildly manic; however, during periods of mania, they may also affect their interactions with their surroundings and may not be able to complete the work tasks given to them by their superiors.

\subsection{Family History}

The Bipolar disorder could be passed through family histories. The research result shows that in a family with a Bipolar disorder patient, there is a high possibility of other members get bipolar disorder [16]. This is not enough to prove that bipolar disorder could pass through genes. However, additional research has brought up clues about the family link between bipolar disorder and genes. There has also been researched on eggs and genes. The study shows that there has a $20 \%$ chance of bipolar disorder pass through genes or eggs [16]. An observation has been put onto a family where the grandpa and the father of the young daughter have been diagnosed as bipolar disorder. The researcher has doubt about the relationship of the bipolar disorder and the family tree [17]. Surprisingly, during the observation, the young daughter has also been diagnosed as having bipolar disorder in a young age.

\section{TREATMENT}

\subsection{Interpersonal and Social Rhythm Therapy}

Interpersonal and social rhythm therapy has its origins in interpersonal psychotherapy for depression [18]. Adults recover more quickly with this therapy [19]. Some theoretical models confirm that patients with bipolar disorder relapse. There are usually three possibilities, the first is non-adherence to medication, the second is disruption of the circadian system, and the third is psychological stress [19]. Interpersonal and social rhythm therapy, on the other hand, is the way to try to 
intervene in relapses. The main targets of this approach are interpersonal stress, circadian rhythm disruption and medication non adherence, which are characteristics shared by adolescents and adults. For adolescents, interpersonal issues are of paramount importance. There are experiments showed that adolescents are very receptive to this approach, and they suggested it can help them become more regular in their social habits and sleep schedules [20]. There's a lot of evidence that people with bipolar disorder have mood instability that's related to circadian variation. The therapy is very effective, with the most significant relapse delays. This treatment option stabilizes the patient's daily life and saves the patient a greater degree of money [21].

\subsection{Cognitive-behavioral therapy}

Cognitive therapy for depression has been applied to patients with bipolar disorder. Patients receiving cognitive-behavioral therapy have somewhat fewer depressive episodes [20]. Few studies have been done that only examine the cognitive-behavioral treatment aspects of bipolar disorder. In today's society, there are many individuals and groups form interventions. They build learning skills and strategies and connections between their patients' daily lives to improve their psychological outcomes, especially in terms of interpersonal interactions and work. However, in recent studies, cognitive therapy has been found to have no significant effect on alleviating cognitive dysfunction in some patients [22].

There is much evidence that non-invasive brain stimulation techniques can play an important role in cognitive function, as it modulates neuroplasticity [22]. This may be a new approach for the treatment of the bipolar disorder. Research on brain stimulation techniques in bipolar disorder is still scarce, so it need more studies to fill this gap for the time being. Intensive psychotherapy is not very effective in the treatment of patients with acute manic episodes. Patients who receive intensive treatment over a period of time recover a little faster than those who receive short-term treatment. Patients who received family-centered treatment had a somewhat lower relapse rate, and the likelihood of their relapse is related to the mood of the caregiver in the family [21].

\subsection{Psychoeducational Intervention}

The purpose of the psycho educational intervention was to provide patients with more information about the disease, thus helping them to better comply with their medication, avoid drug abuse, and relieve their stress and emotions. Studies have shown that patients' perceptions of and dependence on medications are improved by this approach [23]. Thus, psycho education is an adjunct to drug therapy and has great potential for future use.

\subsection{Functional remediation}

Functional remediation is an innovative intervention designed to restore psychosocial functioning in patients with bipolar disorder [22]. By providing a number of everyday neurocognitive approaches and techniques, functional remediation can address the major neurocognitive deficits associated with bipolar disorder. Interventions include individual and group tasks, thus creating a link between the skills and strategies learned and the daily life situation of bipolar disorder. These patients with bipolar disorder showed substantial improvements in overall psychosocial outcomes, particularly interpersonal and occupational functioning. The intervention was also effective for patients with type II bipolar disorder. Analyses revealed that the intervention also improved neurocognitive outcomes in a subsample of patients with cognitive impairment. Interestingly, there were also significant improvements in non-literal memory [22].

\section{PROGNOSIS}

\subsection{Results of treatments}

Bipolar disorder was a life long mental disorder, treatments could ease the symptoms, however, it could not be completely cured. The recurrence rate is high, and the condition of the bipolar disorder patient could become progressively worse if stimulation occurred.

\subsection{Death rate}

Due to the incurable lifelong effect, some patients still consist on following the treatment and alleviate the symptoms. However, a majority of bipolar disorder patient wanted to kill themselves in order to end this torture for both them and people around them. The majority of bipolar disorder patients have suicide attempt. Only some of them succeed, while some others still keep trying to suicide.

\section{CONCLUSION}

This article briefly talked about Bipolar disorder, based on three main aspects. This study has discussed the characteristics of bipolar disorder, the possible cause of bipolar disorder, and the treatment stage of bipolar disorder, and the prognosis of bipolar disorder. Bipolar disorder, as a relatively complex mental disorder, has two main episodes, mania, and depression. The cause of Bipolar is uncertain. Whoever people have testified several aspects of possible influence, such as gender, age, and family history. Even though the rate of successful treatment is relatively low for bipolar disorder, there are still therapies, interventions, medicines used to treat bipolar disorder. The high death rate of bipolar disorder is mainly because of the in-curability and recurrence of bipolar disorder. 


\section{REFERENCES}

[1] WHO., 1. (1993). The ICD-10 classification of mental and behavioural disorders. World Health Organization.

[2] American Psychiatric Association, A. (1980). Diagnostic and statistical manual of mental disorders (Vol. 3). Washington, DC: American Psychiatric Association.

[3] U.S. Department of Health and Human Services. (n.d.). Bipolar Disorder. National Institute of Mental Health. Retrieved from https://www.nimh.nih.gov/health/statistics/bipolardisorder.

[4] Merikangas, K. R., Jin, R., He, J. P., Kessler, R. C., Lee, S., Sampson, N. A.,. \& Zarkov, Z. (2011). Prevalence and correlates of bipolar spectrum disorder in the world mental health survey initiative. Archives of general psychiatry, 68(3), 241-251.

[5] Health.com. (2017, December 7). U.S. Has Highest Bipolar Rate -- Why? HuffPost. Retrieved from https://www.huffpost.com/entry/us-has-highestbipolar-ra_n_833560.

[6] 40 Profound Bipolar Disorder Suicide Statistics. HRF. (2015, February 20). Retrieved from https://healthresearchfunding.org/40-profoundbipolar-disorder-suicide-statistics/.

[7] Judd, L. L., \& Akiskal, H. S. (2003). The prevalence and disability of bipolar spectrum disorders in the US population: re-analysis of the ECA database taking into account subthreshold cases. Journal of affective disorders, 73(1-2), 123-131.

[8] Simon, G. E. (2003). Social and economic burden of mood disorders. Biological psychiatry, 54(3), 208215 .

[9] Goetzel, R. Z., Hawkins, K., Ozminkowski, R. J., \& Wang, S. (2003). The health and productivity cost burden of the "top 10" physical and mental health conditions affecting six large US employers in 1999. Journal of occupational and environmental medicine, 45(1), 5-14.

[10] Leibenluft, E. (1999). Gender Differences in Major Depressive Disorder and Bipolar Disorder. CNS Spectrums, 4(10), 25-33.

[11] Kriegshauser, K., Sajatovic, M., Jenkins, J. H., Cassidy, K. A., Muzina, D., Fattal, O., Smith, D., \& Singer, B. (2010). Gender differences in subjective experience and treatment of bipolar disorder. The Journal of nervous and mental disease, 198(5), 370372.
[12] Martinez·Aran, A., Vieta, E., Torrent, C., Sanchez·Moreno, J., Goikolea, J. M., Salamero, M.,. \& Ayuso-Mateos, J. L. (2007). Functional outcome in bipolar disorder: the role of clinical and cognitive factors. Bipolar disorders, 9(1-2), 103-113.

[14] Simon, G. E., Bauer, M. S., Ludman, E. J., Operskalski, B. H., \& Unützer, J. (2007). Mood symptoms, functional impairment, and disability in people with bipolar disorder: specific effects of mania and depression. The Journal of clinical psychiatry, 68(8), 0-0.

[15] Sachs, G. S.,. \& Grunze, H. (2006). Influence of sub-syndromal symptoms after remission from manic or mixed episodes. The British Journal of Psychiatry, 189(6), 515-519.

[16] Jones, S. (2004). Psychotherapy of bipolar disorder: a review. Journal of Affective Disorders, 80(2-3), $101-114$

[17] Sara Solovitch Last Updated: 18 Aug 2019, \& Solovitch, S. (2019, August 18). Bipolar Disorder \& Genetics: Passed Down Through The Family Tree: Is Bipolar Hereditary? bpHope.com. https://www.bphope.com/unraveling-the-familytree/.

[18] Klerman GL, Weissman MN, Rounsaville BJ, Chevron RS. (1984). Interpersonal Psychotherapy of Depression. New York: Academic Press.

[19] Miklowitz, D. J., Otto, M. W., Frank, E., ReillyHarrington, N. A., Wisniewski, S. R., Kogan, J. N., ... \& Sachs, G. S. (2007). Psychosocial treatments for bipolar depression: a 1-year randomized trial from the Systematic Treatment Enhancement Program. Archives of general psychiatry, 64(4), 419-426.

[20] Hlastala, S. A., Kotler, J. S., McClellan, J. M., \& McCauley, E. A. (2010). Interpersonal and social rhythm therapy for adolescents with bipolar disorder: treatment development and results from an open trial. Depression and anxiety, 27(5), 457-464.

[21] Geddes, J. R., \& Miklowitz, D. J. (2013). Treatment of bipolar disorder. The lancet, 381(9878), 1672 1682.

[22] Solé, B., Jiménez, E., Torrent, C., Reinares, M., Bonnin, C. D. M., Torres, I.,. \& Vieta, E. (2017). Cognitive impairment in bipolar disorder: treatment and prevention strategies. International Journal of Neuropsychopharmacology, 20(8), 670-680.

[23] Jones, S. (2004). Psychotherapy of bipolar disorder: a review. Journal of Affective Disorders, 80(2-3), 101-114. 